\title{
Re-Appraising Instance Seeking in Public Clouds
}

\author{
John O'Loughlin, Lee Gillam \\ Department of Computer Science \\ The University of Surrey \\ Guildford, England \\ \{john.oloughlin, 1.gillam\}@ surrey.ac.uk
}

\begin{abstract}
Variable compute performance has been widely reported on for virtual machine instances of the same type, and price, on Public Infrastructure Clouds. This has led to the proposal of a number of so called 'instance seeking' or 'placement gaming' strategies, with the aim of obtaining better performing instances for the same price for a given workload. However, a number of assumptions made in models presented in the literature fail to hold for real large-scale Public Infrastructure Clouds. We demonstrate, using data from our experiments on EC2, the problems of such assumptions, discuss how these models are likely to underestimate the costs involved, and demonstrate why such literature requires a better Cloud Compute Model.
\end{abstract}

Keywords—performance; clouds; seeking; placement;

\section{INTRODUCTION}

Infrastructure as a Service (IaaS) Clouds allow users to rent resources such as Virtual Machines (VMs) and storage ondemand. These resources can be scaled up and down as required with, in theory, no limit on usage. A metered, pay for what you consume, pricing model provides predictable costs for Cloud consumption. At a minimum, such services free users from having to invest in on-premise resources simply to meet peak demands, as peaks can be offloaded onto Public Clouds. This is beneficial to users for a number of reasons: (1) unused resources from the Public Cloud can be released, so accrue no further cost; (2) required resources can be quickly procured and made operational, meaning expansion when needed is not limited by size or capacity; (3) capital expenditure can be turned into operational expenditure, which is fully deductible in the accounting period in which the expense was incurred.

Successful Infrastructure Clouds are likely to be large to drive the economies of scale, so will almost certainly become heterogeneous over time. As a consequence, VMs of the same type, and price, may well be running on different hardware. This results in variation in their respective performance when running CPU bound tasks - along the lines of that reported elsewhere [1][2]. Variable compute performance in purportedly similar instances (a running VM) means that the amount of 'work' that they can complete per unit of billable time will be different. Equivalently, for a fixed amount of work, differences in performance produce differences in workload execution time, and so potentially difference in costs.

For users running such CPU bound workloads, there is clearly an incentive to be able identify, and obtain, better performing instances of the same type. This has led to various 'instance seeking' or 'placement gaming' strategies being proposed [1][3][4] which aim to do just this. Either analytically, or through simulations, such work attempts to quantify potential performance gains against costs, for different types of strategies that may be employed. Attempts at such quantification rely on a number of assumptions, either explicitly or implicitly made, based on limited experimental data regarding, amongst others: performance distribution and VM allocation policies. Such assumptions should be experimentally verifiable by other users, if not, then results obtained from any models relying on them will need to be reevaluated.

In this paper we present experimental data which we use to examine the validity, and general applicability, of the assumptions made in the literature. Experimental data are collected from Amazon's Elastic Compute Cloud (EC2), where such papers tend to look to verify their approaches. We discover numerous issues that lead to: (1) inaccurate representation of application performance; (2) likely underestimating of instance seeking costs; and (3) Cloud models which do not fully reflect how EC2, and other major Clouds, work.

The rest of this paper is structured as follows: Section II provides background information on Infrastructure Cloud Architecture and the degree of heterogeneity that can be found in today's Clouds. Section III reviews related work, with a brief look at performance variation, followed by an in depth look at work on Instance Seeking to date. In section IV we describe results of performance and resource allocation experiments on EC2, and we use these results to discuss validity and general applicability of extant Instance Seeking work in section V. Finally, in section VI we present or conclusions and discuss future compute modelling work.

\section{BACKGROUND}

Popular Infrastructure as a Service (IaaS) Cloud providers offer access to systems on a very large scale, with sizes of 
individual data centres measured in terms of space at hundreds of thousands of square feet. The ability to quickly scale out, and then back in again, as required, together with a multitude of OS choices and middleware components makes Clouds an attractive platform for a range of uses. In this paper we are primarily concerned with CPU bound workloads, and when we use the term performance we are referring to compute performance, unless otherwise stated.

Examples of CPU bound workloads running on the Cloud, such as Amazon's Elastic Transcoder, a video transcoding service, can be readily found. The Cloud is increasingly used for running batch processing jobs with the popular MapReduce platform. Yahoo, the developers of MapReduce, identify the following tasks as CPU bound: natural language processing (NLP), clustering, classification and graph processing [5]. Finally, and perhaps more surprisingly, CPU bound tasks can also be found in web applications, as Instagram discovered [6]: their web front end, comprising approximately 100 EC2 instances all running the Apache http server and Tomcat java application server, was found to be CPU bound. Appropriate instance type selection was then critical for delivering required performance.

Although global in scale, Clouds do expose a structure which allows users to place instances into specific sub-layers. For example, EC2, the largest Public Cloud by most measures, is organised as a Region/Availability Zone (AZ) model [7]. Regions correspond to geographical locations, such as EUWest-1 (Dublin) and comprise of multiple data centres. Data centres are grouped into AZs, which are physically isolated from each other and have independent power and network connections. In theory, such isolation should prevent problems in one $\mathrm{AZ}$ from escalating to another, and so offer the potential for building infrastructure fault tolerant applications. As of 2012, US-East-1 (N. Virginia), the first Region to come online, consisted of 10 data centres structured into 5 AZs. EC2 Regions are self-contained, independent and share no physical infrastructure, and in this sense they could be thought of as 'sub-clouds'.

Google Compute Engine (GCE) and HP Public Cloud also use the Region/AZ model, again with AZs being isolated locations [8][9]. In common with EC2, users can place instances into any AZs they have access to. Microsoft Azure offers multi-data centre Regions, but not AZs. However, it does allow for a degree of intra-Region isolation via fault domains, which currently consists of a standard 42U server rack [10]. Instances placed in different fault domains have separate power and network connects, although may well be in the same data centre and so are isolated to a lesser degree than AZs. Users cannot place instances into fault domains; instead they assign a role to their instances and the Azure fabric controller will ensure instances with the same roles are dispersed amongst fault domains.

Rackspace [11] also has multi-data centre Regions, but no AZs and no apparent intra-Region isolation mechanisms. Smaller IaaS providers such as GoGrid and Joyent simply advertise a (small) number of geographically dispersed data centres [12][13].
On EC2, an AZ identifier, such as us-east-1a, is a mapping from a user account to an AZ. However, the same identifier may map to different AZs for different accounts. Amazon does not specify whether the mapping allows for use of all possible resources available in an AZ, or to some subset of resources.

Within an AZ, it is possible to request specific kinds of resources, and one such kind is server instance types. Server instance types are specified by fixed sizes with hardware consistency in terms of a given number of vCPUs, some amount of RAM, and some amount of local disk space. For example, any requested m1.medium instance on EC2 [14] will have 1 vCPU, 3.75GB of RAM and 410GB of local storage. Instance types are grouped into classes, or families, and types within the same class are typically 'multiples' of each other, for example, the M1 also contains the m1x.large, which has 4 vCPU, 15GB of RAM and 1640GB of local storage - and so is quadruple the m1.medium. Consequently, instance types within the same class have a fixed ratio of RAM to vCPU, which for the M1 class is 3.75GB per vCPU, whilst instances in the C3 High CPU class have $1.875 \mathrm{~GB}$ per vCPU. Further classes include M2 High Memory, M3 Second Generation General Purpose and $\mathrm{C} 1 \mathrm{High} \mathrm{CPU}$.

In addition to differences in the ratio of RAM to vCPU, instance classes differ by the compute capability of the vCPU. Some providers express compute power in terms of their own devised ratings: on EC2 this is the EC2 Compute Unit (ECU) and on the $\mathrm{C} 3$ class, for example, a vCPU is rated at 3.5 ECUs, whilst on the C1 Class a vCPU is 2.5 ECUs. Google Compute Engine Unit (GCEU) and HP Cloud Compute Unit (HPCCU) also exist but are not readily comparable. More recently, Intel has introduced its Standard Compute Unit (SCU), with the intention of providing homogeneous compute performance across heterogeneous Intel Xeon CPU models [15].

Due, at minimum, to hardware releases, EC2 ends up with multiple hardware platforms supporting instance classes, and some classes end up being supported by several platforms. The platform to class association is sometimes advertised, for example the C3 class only runs on Intel Xeon E5-2680 v2 (Ivy Bridge) Processors. The M3 class however, will run on either an (Intel Xeon) E5-2670 (Sandy Bridge) or an E5-2670 v2 (Sandy Bridge). The E5-2670 v2 is the next generation of the E5-2670 family, and has a higher core count (10 against 8) and a decreased clock rate. The M1 class has at least 6 hardware platforms supporting it [16]. Such associations are true at the time of writing, but will likely expand in due course.

The various hardware platforms lead us to use the term heterogeneous specifically to refer to those platforms that support the same instance class, rather than referring to supporting different instance classes. In this sense, the M3 class became heterogeneous with its second hardware platform; when first made available it ran only on the E5-2670. The M1 class is certainly heterogeneous, but the extent of this is not advertised and we have had to determine the associations ourselves, identifying the CPU (using/proc/cpuinfo on Linux) across a large number of instances run across EC2; Table 1 shows associations we determined in an 18 month period from March 2013 to September 2014 for around 9000 instances, as well as some which are advertised. 
TABLE I. INSTANCE CLASS TO CPU MODEL ASSOCIATION

\begin{tabular}{|l|r|}
\hline \multicolumn{1}{|c|}{ Class } & \multicolumn{1}{c|}{ CPU Models (Intel Xeon unless otherwise stated) } \\
\hline M1 & E5430, E5645, E5507, E5-2650, E5-2651 and AMD 2218 HE \\
\hline M2 & X5550 and E5-2665 \\
\hline C1 & E5345, E5410, E5506 and E5-2650 \\
\hline M3 & E5-2670 and E5-2670 v2 \\
\hline C3 & E5-2680 v2 \\
\hline
\end{tabular}

In addition to differences in CPU model, there may also be differences in various other hardware subsystems in hosts with the same CPU, but in general these are hard, if not impossible, to detect.

\section{RELATED WORK}

The heterogeneity shown above has been reported in a number of papers, and it has been identified as the major cause of performance variation on EC2. Armbrust et al. [17] describe EC2 performance as unpredictable whilst Osterman, et al. [18] describe performance as unreliable, Yelick, et al. [19] found, simply, that "applications in the Cloud...experience significant performance variations" in part caused by heterogeneity (of EC2); Phillips, Engen, and Papay [2] discovered differential performance in instances of the same type on EC2 when attempting to predict application performance. It is important to qualify such statements with respect to 'significant' or at least 'differential' to understand the extent of these, and in particular with respect to prediction for specific applications.

In respect to Regions, Schad, et al. [20] showed that compute performance in US East N. California and EU West Dublin falls into two distinct performance bands which, upon further investigation breaks out by the two different CPU models backing their instances. Since we have seen rather more than two CPU models, this also demands further investigation. Further, no per AZ differences were described.

In [1][3][4] the authors explore so called 'instance seeking' or 'placement gaming' strategies, which attempt to exploit performance differences of same priced instances by searching for and deploying best performing instances, whilst ditching worse performing ones. We describe these in more detail below.

\section{A. Paper 1: Exploiting heterogeneity within the same \\ Instance type on EC2}

In [1], the authors use unixbench to measure CPU performance of m1.large instances in US-East-1 (N.Virginia). Unixbench runs a number of tests, each one of which is normalised against a fixed reference machine, and the geometric mean of these is then taken to produce a composite score. Three CPU models were identified: E5430, E5645 and
E5507, and they benchmarked 20 of each type - so 60 in total. Intra CPU model results showed small variation, leading the authors to regard it as negligible. Each CPU type is given a unixbench score, referred to as a performance level, calculated as the average of all the unixbench scores for the given CPU. They compare inter CPU performance by use of a multiplier from the score of the lowest performing CPU. The CPU with the lowest score was the E5507, and the E5430 was 1.1 times better, whilst the E5646 was 1.21 times better. Hence their indexed score, with respect to the E5507 baseline is 1, 1.1 and 1.21 .

The proportions of CPU models obtained from requests to US-East-1 are used to estimate of the probability of obtaining a model with a given CPU type. Their Cloud model, although not explicitly described as such, is this: a Cloud consists of unlimited numbers of instances; the probability of obtaining an instance with given a CPU is fixed and independent of the CPU models obtained by other instances. The performance of an instance is the indexed score associated with the CPU model backing an instance, as described above.

Their strategy for obtaining better performance is simply: start a number of instances and determine from the CPU model which are the best. Deploy these instances and ditch the rest. Start the same number of ditched instances and test for desired CPU model again. Iterate until you have obtained the required number of instances with the desired CPU.

They claim that such an approach could lead to a $30 \%$ cost reduction over the course of a year in the case where the best performing instances are scarce.

\section{B. Paper 2: More for your Money: Exploiting Performance Heterogeneity in Public Clouds}

The above is an example of a Grey-box strategy, as selection decisions are based entirely on CPU. A Black-box strategy is one where a set of instances are benchmarked at start up, comparisons are made between those instances, and instance selection is based entirely on those comparisons. In [3] various Grey-box and Black-box strategies are described: $C P U, C P U-M, C P U-M A X, P E R F$ and PERF-M - 3 grey box and 2 black box respectively.

The term $(\mathrm{A}, \mathrm{B})$ strategy, where $\mathrm{A}$ and $\mathrm{B}$ are integers, is used to mean that $\mathrm{A}$ instances will run for $\mathrm{T}$ units of billable time, and that B instances will run for 1 unit of billable time each. Hence the cost for each strategy is the same: AT + B. This differs from paper 1 as they fix the number of extra instances to run to $\mathrm{B}$.

The CPU and PERF strategies are similar. Both start A + B instances. The CPU strategy deploys the best A instances, as determined by CPU model, ditching the remaining $\mathrm{B}$. The PERF strategy benchmarks all A + B instances, deploys the best $\mathrm{A}$, as determined by the benchmarking, and ditches the remaining $B$. We have $A+B$ instances running in the first hour, with $\mathrm{A}$ instances running in the remaining T-1 hours. All selections, either CPU or performance based, complete in the first hour, which the authors refer to as 'up front' determination. 
CPU-M and PERM-M strategies use a mixture of 'up front' determination and 'opportunistic replacement'. PERF-M starts $\mathrm{A}+\mathrm{B}$ instances and calculates the mean performance of the set. At the end of the first billable hour B instances are shutdown, in accordance with the CPU and PERF strategies. In each of the subsequent T-1 billable hours (where there is an A instance running) they benchmark all running instances, and shutdown any instances whose performance is sufficiently poor as compared to the average calculated in the first hour. These instances are replaced by starting the same number of new instances. The process of benchmarking and terminating poorly performing instances and replacing with new ones is referred to as 'opportunistic performance replacement'.

CPU-M is similar to PERF-M, and starts by running a CPU strategy on $\mathrm{A}+\mathrm{B}$ instances in the first hour, and then using opportunistic performance replacement in the subsequent T-1 hours. Finally, the CPU-MAX strategy starts the same as the CPU strategy, but in the second hour onwards seeks to replace any of the A instances without the best CPU.

Their experimental work is based on the benchmarking of $39 \mathrm{~m} 1$.small instances and for compute benchmarking they use NQueens, mcf and sphinx3, the latter two being part of SPEC CPU 2006 suite. They measure execution times, and calculate a speed-up against the worst performing. For the SPEC benchmarks they find more intra CPU variation then they do for NQueens, which leads them to consider a performance distribution per CPU model for each of the applications under consideration.

Their Cloud model is the same as in paper 1, except for performance: A Cloud consists of unlimited numbers of instances; the probability of obtaining an instance with a given CPU is fixed and independent of the CPU models obtained by other instances. The performance of an instance backed by a given CPU (with respect to a given application) is assumed to be normally distributed; instances with different CPUs model will have different means but the same standard deviation.

By changing the means and standard deviations they can consider various scenarios such as (1) application performance with negligible intra CPU variation, and wide performance differences across the CPU models or (2) applications with overlapping CPU performance and large intra CPU variation. Each simulation would therefore consist of the following parameters: A, B, means per CPU, standard deviation, $\mathrm{T}$ and a particular strategy. Rather than using empirically derived performance data, they fix $\mathrm{A}=10, \mathrm{~T}=24$ and then explore various values of these parameters to compare strategies.

We describe the models above as Opaque Cloud Models, as they model the observed interaction with a Cloud system, and do not include descriptions of how a Cloud system operates internally.

\section{Paper 3 Impact of Instance Seeking Strategies on Resource Allocation in Cloud Data Centres}

In follow-on work from paper 1 , the authors examine the effect of instance seeking on Cloud resources in [4]. An Opaque Cloud model is now insufficient as the 'internal' operations of the Cloud need to be incorporated into the model.
The author's base their model on the one described in [21], which in turn is guided by Eucalyptus Private Cloud architecture [22].

In this model, Clouds consist of data centers which are comprised of 'clusters' of hosts. Note that the term 'cluster' as used here implies nothing beyond a 'group' of hosts. It is not explicitly stated though whether a cluster is intended to represent a physical entity, such as a fault domain found in Azure Cloud, or a logical entity such that the membership is software defined.

The clusters are considered to be different sizes and also to be heterogeneous, that is, to contain instances backed by a range of CPU models, and with different models having different probabilities of being obtained. Further, for a given CPU model the probability of obtaining it differs across the clusters.

There is no ability for a user to place an instance in a specific cluster. VM allocation polices are used to specify how an instance request is allocated to a physical host. In this case a 2 stage allocation process exists: an instance is first allocated to a cluster, and then allocated to a host within the cluster. Three cluster allocation policies are considered - random, round robin, and least loaded - together with 2 host allocation polices - random and 'least core full'. In total this gives 6 polices.

Updated and additional instance seeking strategies are then presented - but are only considered to be of use to 'performance oriented users'. These are users for whom performance is more important than costs, and the instances are intended to run for a long time. The moderate strategy is an update on seeking for one CPU model, and allows the user to set a threshold performance value, and by using this value the user can identify 'worst' or 'best' instances. The performance threshold determination proceeds via CPU model identification. As before, the user deploys 'best' instances, ditches 'worst' instances, and starts new ones to replace the ditched instances. The process is iterated until the required number of instances with the desired CPU model is found.

In the 'moderate' strategy, users only request precisely the number of instances they require. For example, if a user requires 15 'better' instances but after a request only has 10 of the best and 5 of the worst, they terminate the 5 worst and a request for 5 more instances is made. This process is then iterated until the required number of instances with the desired CPU is obtained.

In a greedy strategy a user will always request more instances than are actually needed until they find the required number with the desired CPU model. The exact number requested depends upon how close they are to required number, and is defined by a piecewise 'greedy' function. Again, this is iterated until the required number of instances with the desired CPU model is obtained. Finally, in a collaborative strategy multiple users work together to increase their chances of obtaining 'better' instances. They do this by releasing back better instances when not needed, so increasing the chances that others may obtain them.

In their simulations they consider a data centre with 3 clusters, and each cluster contains instances backed by one of 
the following Intel Xeons: E5430, E5507 or E5645, and performance data for instances is as described in [1]. Under various circumstances, the authors claim to show how a cluster can reach an exhaustive state i.e. with very few 'better' instances left, and that users employing a greedy strategy can impact users deploying either a moderate strategy or using no strategy.

As this model considers data centre, clusters, allocation policies, and numbers of hosts, we refer to it as a SemiTransparent Cloud model.

\section{COMPUTE AND RESOURCE EXPERIMENTS ON EC2}

In this section, we present results from three experiments performed on EC2.. The first is a performance experiment conducted in May - June 2014 and first reported in [16]; the second is an EC2 resource allocation experiment conducted in January 2014; and the third is a co-location performance experiment conducted in October 2014.

\section{A. Experiment 1: EC2 Compute Performance}

In this experiment we ran $\sim 500 \mathrm{~m} 1$.small instances in 7 AZs in the Regions US-East-1 and EU-West-1. Each instance ran 5 benchmarks: 4 compute benchmarks and one memory bandwidth benchmark. The compute benchmarks were: bzip2, POV-Ray, GNU GO and NAMD, whilst the memory bandwidth benchmark was STREAM; and we describe these in more detail below:

- The bzip2 benchmark is a measure of CPU integer performance, and is part of the Standard Performance Evaluation Corporation (SPEC) CPU integer benchmark suite, SPECint 2006 [23]. The input file was an ubuntu 10.04 desktop ISO of size 694MB.

- The POV-Ray benchmark [24] measures floating point performance, and is part of the SPEC floating point suite, SPECfp 2006 [25]. POV-Ray is provided with a scene description file, benchmark.pov, from which it uses ray tracing to produce a photo realistic image.

- The GNUGO [26] benchmarks measure CPU integer performance by playing the Go game. Go is provided with a SGF input file and plays the game until completion. It is part of the SPECInt 2006 suite.

- NAMD [27] is a bio-molecular simulation, using the standard input of apoa1.namd. It measures floating point performance and is part of SPECfp 2006.

- STREAM [28] measures memory bandwidth, that is, the $\mathrm{MB} / \mathrm{s}$ that can be transferred from main memory to the CPU. The benchmark performs a number of linear operations on vectors, the size of which must be chosen so as to ensure results are too large to be cached in the L2 cache.

In Table II below we present summary statistics.
TABLE II. SUMMARY STATISTICS

\begin{tabular}{|c|c|c|c|c|c|}
\hline & $\begin{array}{c}\text { CPU } \\
\text { model }\end{array}$ & mean(s) & sdev & skew & kurtosis \\
\hline \multirow[t]{6}{*}{ Bzip2 } & E5430 & 447 & 14.4 & 0.44 & 0.14 \\
\hline & E5-2650 & 469 & 14.3 & 1.05 & 1.25 \\
\hline & E5-2651 & 478 & 19.7 & 0.48 & 1.77 \\
\hline & E5645 & 504 & 22.9 & -2.29 & 4.66 \\
\hline & E5507 & 649 & 40.4 & 0.64 & -0.57 \\
\hline & AMD 2218 & 684 & 17.8 & -0.32 & -1.09 \\
\hline \multirow[t]{6}{*}{ GNUGO } & E5430 & 199 & 2.8 & 1.26 & 2.56 \\
\hline & E5-2650 & 207 & 2.8 & 0.86 & 1.6 \\
\hline & E5-2651 & 213 & 7.3 & 0.18 & -.0 .08 \\
\hline & E5645 & 180 & 8.4 & -2.75 & 6.14 \\
\hline & E5507 & 199 & 2.6 & 0.88 & 0.23 \\
\hline & AMD 2218 & 191 & 1.27 & -0.31 & -1.29 \\
\hline \multirow[t]{6}{*}{ POV-Ray } & E5430 & 580 & 2.9 & 44 & 3.46 \\
\hline & E5-2650 & 556 & 5.6 & 2.1 & 5.29 \\
\hline & E5-2651 & 564 & 9.9 & 0.18 & -0.45 \\
\hline & E5645 & 514 & 25.3 & -2.88 & 6.57 \\
\hline & E5507 & 545 & 4.9 & 0.97 & 0.37 \\
\hline & AMD 2218 & 448 & 2.5 & 0.49 & -0.92 \\
\hline \multirow[t]{6}{*}{ NAMD } & E5430 & 2160 & 20.7 & 0.59 & 1.02 \\
\hline & E5-2650 & 2007 & 28.5 & 0.78 & 1.01 \\
\hline & E5-2651 & 1994 & 41.9 & -0.02 & -0.48 \\
\hline & E5645 & 2043 & 96.4 & -2.8 & 6.4 \\
\hline & E5507 & 2187 & 18.1 & 0.26 & 0.045 \\
\hline & AMD 2218 & 2416 & 7.3 & 0.22 & -0.94 \\
\hline \multirow{6}{*}{$\begin{array}{l}\text { STREAM } \\
(\mathrm{MB} / \mathrm{s})\end{array}$} & E5430 & 3335 & 320 & -4.08 & 23.18 \\
\hline & E5-2650 & 7568 & 382 & -10.1 & 116.88 \\
\hline & E5-2651 & 4753 & 572 & -0.59 & -1.3 \\
\hline & E5645 & 5128 & 1332 & -1.2 & -0.42 \\
\hline & E5507 & 4779 & 210 & -1.27 & 2.88 \\
\hline & AMD 2218 & 1957 & 744 & -0.33 & -2.04 \\
\hline
\end{tabular}


For each task we can order (from best to worst) the CPUs by their mean performance, as show in Table II. For the bzip2 task we have E5430, E5-2650, E5-2651, E5645, E5507 and AMD 2218. The CPU mean ordering is task dependent; that is, the ordering can be different for each task. Interestingly, even comparing the integer benchmarks (bzip2 and GNUGO) we have quite different orderings, and similarly for the floating point benchmarks.

In general, this means there is no 'best performing CPU model' for all applications. Interestingly, it is also difficult to infer CPU performance simply from age: the E5430, the second oldest model, is the best performing for bzip2. The E52651, the latest model, is the worst model for GNUGO.

There can be large variation in a CPUs performance, as we can see by observing the range for E5507 on bzip2. There can also be considerable overlap in performance for different CPU models. Performance is generally skewed, and compute performance is typically positively skewed. This indicates there are more examples of instances with worse performance (higher execution times), and we find few examples of 'performance bursting'. For STREAM, we find negative skew; so again, more examples of worse performance (lower MB/s).

The performance characteristics of the E5645 are somewhat different to the other models. This CPU exhibited a large standard deviation for all the tasks (relative to the other models), as well as being negatively skewed for compute performance. This is explained by there being a small group of instances backed by the E5645, in eu-west-1a, whose performance was significantly better than other E5645. Removing these instances from the E5645 set, the remaining models have similar characteristics to the other model types. Having examined the /proc/cpuinfo and /proc/meminfo files from these instances, we could find no differences that would explain the improved performance for this small group alone.

In general, CPU models are delivering performance in ranges. For example, for bzip2 the E5-2650 always delivered in the range $442 \mathrm{~s}$ to $515 \mathrm{~s}$. This consistency is important, as it allows for per CPU model performance predictions, within given ranges, to be made. For certain tasks, such as POV-Ray, the coefficient of variation (defined as the ratio of standard deviation to the mean) and performance range is quite small; whilst for bzip2 this range can be quite large, and indeed consistently so - particularly for the E5507.

There are a number of possible explanations for the size of the range in bzip2 performance on the E5507: (1) variations in the disk subsystems, or indeed other hardware, of the E5507 hosts, and such variations cannot be readily investigated in the data collected; (2) I/O contention amongst the instances running on the hosts, which for reasons we cannot observe is more problematic with the E5507 hosts; (3) the size of the L2 cache, as compared to the other hosts, is low and this may well be significant for bzip2. On the E5507 we have 1MB per core of L2 cache, whilst the highest ratio is $3 \mathrm{MB}$ per core, found on the E5430, and if we order the CPU models by this ratio we find the same ordering as for the bzip2 task.

\section{B. Experiments 2 and 3: EC2 Resource Allocation}

Two resource allocation experiments were conducted in January and October 2014 in us-east-1a (N.Virginia Region) and one in ap-southeast-2b (Sydney Region) respectively. A request for 20 instances was made, the CPU models found were recorded and the instances were released. This was done 5 times in N.Virginia over a 24 hours period, and 10 times in Sydney over the course of a week.

In Sydney all instances were backed by either E5645 or the E5-2650, whilst in N.Virginia we have instances backed by E5645, E5507 or the E5-2650. Tables III and IV below show how many of each type we find in the request. As we can see, there is variation in the number of each CPU model obtained per request, and a Cloud model should be able to account for this variation

TABLE III. RESOURCE ALLOCATION AP-SOUTHEAST-2B

\begin{tabular}{|c|r|r|r|r|r|r|r|r|r|r|}
\hline Sample & 1 & 2 & 3 & 4 & 5 & 6 & 7 & 8 & 9 & 10 \\
\hline E5645 & 5 & 7 & 7 & 5 & 3 & 9 & 8 & 5 & 7 & 12 \\
\hline E5-2650 & 15 & 13 & 13 & 15 & 17 & 11 & 12 & 15 & 13 & 8 \\
\hline
\end{tabular}

TABLE IV. RESOURCE ALLOCATION US-EAST-1A

\begin{tabular}{|c|r|r|r|r|r|}
\hline Sample & 1 & 2 & 3 & 4 & 5 \\
\hline E5645 & 12 & 15 & 11 & 11 & 17 \\
\hline E5-2650 & 6 & 0 & 3 & 6 & 0 \\
\hline E5507 & 2 & 5 & 3 & 3 & 3 \\
\hline
\end{tabular}

\section{CRITIQUE OF INSTANCE SEEKING WORK}

In this section, we re-examine the papers discussed in detail in section III, in light of the experimental results obtained.

\section{A. Performance}

An accurate description of performance should be a priority for any user seeking better performance, particularly so if PERF strategies are being employed.

In paper 1 (the results of which are used in paper 3) intra CPU model performance is negligible, and so the performance of an instance is one of $k$ values, determined by the $k$ different CPU models backing it. This description does not fit with our findings. Histograms of benchmark execution times in section IV show multi-modal continuous distributions. Unixbench [29] is used to measure CPU performance, although we note that the unixbench project states ' $d o$ be aware that this is a system benchmark, not a CPU, RAM or disk benchmark'.

Unixbench is a composite benchmark, and the CPU components are the well known Dhrystone [30] and Whetsone [31] benchmarks. These are synthetic benchmarks, designed to statistically mimic the execution of different instruction types of numerical codes used at the time. On modern CPUs these benchmarks have numerous shortcomings, one of which is that they comfortably fit into the L1 instruction cache. Consequently, they are shielded from variable access times to the memory hierarchy, which are particularly problematic in shared tenancy. We would therefore suggest that the negligible intra CPU variation suggested is merely a function of the 
benchmarks chosen, and should not be considered a characteristic of compute performance more generally.

Paper 2 considers execution times to be normally distributed. This is more inline with our results, and they also note that the degree of intra CPU variation (for a given benchmark) differs across the different CPUs. Again, this is in agreement. However, we do question whether a normal distribution is the most appropriate. On EC2 we find distribution of execution times (for the CPU benchmarks) to be positively skewed, whilst the memory benchmark (STREAM) is negatively skewed, and we find more examples of instances with worse performance. As such, they are not normally distributed, and indeed such an assumption will lead to over estimating the number of good instances and under estimating both the number of bad instances, and how far (in terms of standard deviations) they are from the mean.

An analogy could be drawn between this underestimation of poor performance, performance risk, and the underestimation found in financial risk models, such as Value at Risk (VaR). VaR calculates the maximum loss on a portfolio over a given time period, within a given confidence level. When using the variance-covariance method for VaR calculation, stock returns are assumed to be normally distributed. This assumption leads to an underestimation of large deviations (4 or more standard deviations from the mean) in stock returns, as historical data shows. In turn, this leads to an underestimation in risk to the portfolio as a whole. VaR-x is VaR analysis with a so-called 'fat tail' distribution - whereby such deviations are more likely, and we believe a 'fat tail' distribution is more likely than the normal to describe performance on many Cloud systems, as we now discuss.

EC2, along with Rackspace and numerous other large providers, uses the Xen hypervisor [32]. Xen is responsible for scheduling CPU time to an instance's vCPU(s), similar to how an OS schedules CPU time for processes. In order to fairly schedule vCPUs of the same rating, Xen uses a combination of shares and limits. Shares are a weighting scheme whereby vCPUs with the same weight should receive the same amount of CPU time. The actual CPU time a vCPU will receive will vary according to the total number of vCPUs running. By limiting the total number of vCPUs that can run on a host, together with an equal weighting amongst them, we set a minimum CPU time for vCPUs. In this case then, a vCPU has a minimum but no maximum set. The ability to obtain extra CPU time when it is available is known as 'CPU bursting'. Amazon has recently introduced an instance type that has a guaranteed minimum CPU compute, with an ability to burst [33]. For other instances types, a limit is set - and an instance cannot burst beyond this. We should therefore not expect to see examples of instances with performance beyond this - as would be implied by a normal distribution.

The contention for resources that cannot be partitioned, such as memory caches and buses, has been identified as causing degradation of compute performance. This is known as the 'noisy neighbor' effect. Recent work by Google [34] attempts to identify noisy neighbors and kill the associated processes. The observed skew is most likely, though not definitely, caused by noisy neighbors, and intriguingly, depending on the VM allocation policy being used, it is entirely possible that a user is their own noisy neighbor when their VMs are scheduled onto the same host. Again, we see an analogy between correlation risk in portfolios and performance risk in sets of co-resident instances. In our view the noisy neighbor affect should be included in a Cloud model.

Finally, we have shown that the ordering of CPU by mean performance is application dependent i.e. is different for different applications. In paper 3 they consider the impact of users seeking the 'best' CPU on users who engage in no strategies - and describe resource starvation in this case. However, when application dependency is considered, the impact of one group of user's actions on others is not so clear. For example, a POV-Ray user seeking and obtaining AMD 2218 will potentially benefit a bzip2 user engaged in no strategy, as it will remove one worst CPU model (instance) for bzip2 from the available resources. Realistic simulations should allow for users running a multiplicity of applications needs, each of which may have different CPU performance ordering.

\section{B. Opaque Cloud Models}

The Opaque Cloud Models presented in papers 1 and 2 both consider the Cloud to consist of an unlimited number of instances, where the probability of obtaining an instance with a given CPU is fixed and independent of the CPU models obtained by other instances. They differ in their modeling of instance performance, as described already.

Consider, then, a user seeking a particular CPU, say the E5645. In a request for 20 instances, how many of them are likely to receive a E5645? Let X be the number of E5645 obtained in a request. We know that for this model the probability, say $p$, of obtaining a given CPU model is fixed, and since we have independence: $X \sim \operatorname{Bin}(20, p)$. It is natural to ask if this model generates data of the type we have described in section IV.

In our first example, from Table III, of the 200 instances started in 10 batches of 20 we find the proportion of E5645 to be 0.34 and so we estimate the probability of obtaining an instance backed by a E5645 as 0.34 (so X Bin(20,0.34)). Then $\mathrm{E}[\mathrm{X}]=6.8$, and indeed in 8 of the samples we have $\mathrm{X}$ within 2 standard deviations of the mean. However, by calculation, $\mathrm{P}[\mathrm{X}=3]=0.038$ and we would expect to see this value in (less than) one in 25 samples, whilst $\mathrm{P}[\mathrm{X}=12]=0.01$, and so we would see this value one in a 100 . From this small set of samples, we can see bunching around the mean but with more variation in $\mathrm{X}$ than one would expect to see if $\mathrm{X}$ was binomial.

In our next example from us-east-1a, and presented in Table IV, we had 97 instances - obtained in 4 batches of 20 and one of 17. Of these, 15 are E5-2650, and so we estimate the probability of obtaining an E5-2650 as $\mathrm{p}=15 / 97=0.15$. This time, let $X=$ number of E5-2650 obtained in a request for 20 instances, and so $X \sim \operatorname{Bin}(20,0.15)$. In our 5 samples we have $X=0$ and $X=6$ twice, and from a direct calculation using the Binomial, we find the probability of obtaining either $\mathrm{X}=0$ or $\mathrm{X}>6$ is less than 0.05 in both cases. We should see 
such values for $\mathrm{X}$ less than one in 20 requests, suggesting that such a model fails to adequately describe how CPU models have been obtained in this case.

In general, these examples show that we find more variation in $\mathrm{X}$ than the binomial distribution allows for, and these Opaque Cloud Models do not fully capture the extent of the variation that can be found. Such variation needs to be fully accounted for, as there is now a larger range (smaller minimum and greater maximum) in the number of instances that need to be started in order to find the required number with the desired CPU model. Consequently there is also a greater range in instance seeking costs: if a user is lucky, they may obtain them quickly, but if they are unlucky the costs may outweigh the gain.

\section{Semi-Transparent Cloud Models}

The work in paper 3 attempts to understand potential effects of instance seeking on Cloud resources. In this case then an Opaque Cloud Model is insufficient, and Cloud infrastructure needs to be considered. This includes, at a minimum, Cloud partitioning such as Region/AZ, instance types, hosts types and when applied to specific Clouds, such as EC2, may included CPU models available, their distribution and probability of an instance obtaining one.

The emergence of Private Cloud systems such as Eucalyptus, and more lately OpenStack [35], has provided researchers with some insights into Cloud architecture particularly so in the case of OpenStack which supports a number of large scale Public and Private deployments, including that of HP's Helion. Eucalyptus was used in [22] to analyse VM allocation policies, and paper 3, following on from this, describes Clouds in an essentially identical fashion. As already discussed in section III, we have a model of a Cloud data centre, which comprises a number of heterogeneous 'clusters', and VM allocation is a 2-stage process: allocate to cluster and then to host. Crucially, users do not have the ability to place instances in specific clusters.

We believe this to be a misinterpretation of how clusters in Eucalyptus work. Eucalyptus included an implementation of EC2 API, and a cluster is used to represent an AZ. In Eucalyptus a user can always place an instance into an AZ. Further, we note that the data centre/cluster model described here does not fit how EC2, GCE or HP Cloud works, as in all of these Clouds operate a Region/AZ model and users can place instances into an AZ.

As Clouds expand, different locations within will inevitably contain either different resources or the same resources but in different proportions. For example, we have already seen in this paper that us-east-1a returned E5645, E5507 and E5-2650 whilst ap-southeast-2b returned only E5645 and E5-2650. Further, in ap-southeast-2b, 66\% of instances were backed by the E5-2650 whilst in us-east-1a it was only $15 \%$. Users, who are seeking E5-2650, possibly for POV-Ray or bzip2 work, would choose to place instances in ap-southeast-2b over us-east-1a. Similarly, a user seeking E5645, perhaps due to memory bandwidth performance (as measured by STREAM), would choose us-east-1a over apsouthease-2b. And so, any model which precludes such instance location placement, does not accurately describe how users can, and would, seek instances on Public Clouds. Indeed, we consider location determination as a crucial first step in any instance seeking strategy.

\section{Strategies Employed}

In paper 3 the performance strategy is aimed at 'performance oriented users', where such users are not interested in costs and may run instances for a long time 'potentially forever'. Whilst this may describe users with an infrequently changing infrastructure, we would argue that there are many more use cases that need considering. In particular, users with 'bursty' needs, for example those running applications that need to scale out and back in, in response to changes in demand. Similarly, users who seek to regularly offload (nightly or weekly) batch processing jobs onto the Cloud also have 'bursty' needs.

Paper 2 fixes the cost of finding the extra instances, but also fixes the time horizon at 24 hours. This, then, is asking a somewhat different question: how much performance can be gained for a fixed cost.

In paper 3, where the impact of employing particular strategies on the ability of others to find good instances is studied, we note that user behaviour, in terms of strategies employed, do not change. We consider that an evolutionary approach that allows for adaptations in behaviour would be likely to produce simulations more in line with likely user behaviour.

Finally, we also note the PERF strategies assume no performance history is available for comparison. Although this allows for a degree of flexibility in applying the strategy, we would consider users who employ such strategies would be likely to retain past performance history for comparisons.

\section{CONCLUSIONS AND FUTURE WORK}

Compute bound workloads are prevalent on Public Clouds, and indeed, as Instagram found, these may even be found in some surprising places. The scale that Public Clouds operate at, which is necessary to drive the economies, means that instances of the same type will, over time, be backed by different hardware. This seems inevitable, unless instance types also start to move with the hardware. Consequently, differential compute performance of types seems inevitable at least with Clouds built on the x86 architecture.

This has led to a number of authors proposing various strategies to exploit performance variation by finding better performing instances at the same price. However, to date, the results obtained have been from models which contain certain flawed assumptions, and which therefore have limited applicability to real Public Clouds.

Our intention is to build on and extend findings presented in this paper by developing a new Cloud Compute Model (CCM). This new CCM will incorporate a more nuanced view of compute performance and generate resource distributions inline with those described in section IV, B. Using this, we can then re-evaluate strategies, identify heuristics for adapting instance seeking behavior, and propose new strategies to fully 
explore the relationship between performance gains and associated costs for real Public Clouds.

\section{REFERENCES}

[1] Z. Ou, H. Zhuang, J.K. Nurminem, A. Yla-Jaaski and P. Hui, "Exploiting Hardware Heterogeneity within the same instance type of Amazon EC2," presented at $4^{\text {th }}$ USENIX Workshop on Hot Topics in Cloud Computing, Boston, MA. Jun. 2012.

[2] Phillips, V. Engen and J. Papay, "Snow white clouds and the seven dwarfs," in Proc. of the IEEE International Conference and Workshops on Cloud Computing Technology and Science, pp738-745, Nov. 2011.

[3] B. Farley, et el, "More for yor money: explotiting performance heterogeneity in Public Clouds", in Proc. of the Third ACM Symposium on Cloud Computing, artice no. 20, 2012.

[4] H. Zhuang, X. Liu, Z. Ou and K. Arberer, "Impact of Instance Seeking Strategies on Resource Allocation in Cloud Data Centres", in Proc. Of the IEEE Sixth International Conference on Cloud Computing, pp 2734, 2013.

[5] Yahoo Developer Network. "Benchmarking and Optimising Hadoop," $\begin{array}{lll}\text { www.slideshare.net. } & \text { [Online]. }\end{array}$ http://www.slideshare.net/ydn/hadoop-summit-2010-benchmarking-andoptimizing-hadoop, [Accessed: $30^{\text {th }}$ October 2014].

[6] Facebook. "Instagram Architecure," [Online]. Available: https://www.facebook.com/notes/sebunya-robert/instagram-and-how-itwas-built-i-mean-the-architecture-underneath/10203657075285323 [Accessed: 30th October 2014].

[7] AWS. "Regions and Availability Zones," [Online]. Available: http://docs.aws.amazon.com/AWSEC2/latest/UserGuide/using-regionsavailability-zones.html [Accessed: $27^{\text {th }}$ July 2014].

[8] GCE. "Google Cloud Platform," cloud.google.com [Online]. Available: https://cloud.google.com/compute/ [Accessed: $30^{\text {th }}$ October 2014].

[9] HP. "HP Helion Public Cloud," www.hpcloud.com [Online]. Available:http://www.hpcloud.com/ [Accessed: 30th October 2014].

[10] Technet. "Yung Chou on Hybrid Clouds," blogs.technet.com [Online]. Availabhttp://blogs.technet.com/b/yungchou/archive/2011/05/16/windo w-azure-fault-domain-and-update-domain-explained-for-it-pros.aspxle: [Accessed: 30th October 2014].

[11] Rackspace. "The Rackspace Managed Cloud," www.rackspace.com/cloud. [Online]. Available: http://www.rackspace.co.uk/cloud [Accessed: 30th October 2014].

[12] Joyent. "High Performance Cloud Computing," www.joyent.com. [Online]. Available: https://www.joyent.com/ [Accessed: $30^{\text {th }}$ October 2014].

[13] GoGrid. "GoGrid," www.gogrid.com. [Online]. Available: http://www.gogrid.com/ [Accessed: $30^{\text {th }}$ October 2014].

[14] AWS. “Amazon EC2 Instances,” aws.amazon.com. [Online]. Available: http://aws.amazon.com/ec2/instance-types/ [Accessed: 27th July 2014].

[15] Intel. "Meet Cloud Performance Goals with Intel SAA," www.intel.com. [Online].

Available: http://www.intel.com/content/www/us/en/software/intel-saaperformance-white-paper.html [Acessed: 30th October 2014].

[16] J. O'Loughlin and L. Gillam, "Towards performance prediction for Public Infrastructure Clouds: an EC2 case study," Proc. IEEE Fifth
International Conference on Cloud Computing Technology and Science (CloudCom 2013), Dec2013, pp. 475-480.

[17] M Armbrust, et al, "Above the clouds: a Berkely view of cloud computing”. Technical Report EECS-2008-28, EECS Department, University of California, Berkeley.

[18] S. Osterman et al, "A performance analysis of EC2 cloud computing services for scientific computing," Cloud Computing, Lecture Notes of the Institute for Computer Sciences, Social-Informatics and Telecommunications Engineering, vol 34, 2010, pp 115-131.

[19] K. Yelick, et al, "The Magellan Report on Cloud Computing for Science". [Online]. Available at: http://www/alcf.anl.gov/magellan [Accessed at: 2nd January 2014].

[20] J. Schnad, J. Dittrich, and J. Quiané-Ruiz, "Runtime measurements in the cloud: observing, analyzing, and reducing variance." In, Proc. of the VLDB Endowment 3, no. 1-2,pp 460-471. (2010).

[21] K. Mills, J. Filliben, and C. Dabrowski, "Comparing VM placement algorithms for on-demand Clouds", in Proc. Of the 2011 IEEE Third International Conference on Cloud Compting and Science”, pp 91-98, 2011.

[22] Eucalyptus. "Eucalyptus," www.eucalyptus.com. [Online]. Available: https://www.eucalyptus.com/ [Accessed: $30^{\text {th }}$ October 2014].

[23] SPEC. "SPEC CPU 2006," www.spec.org. [Online.] Available: http://www.spec.org/cpu2006/CINT20006 [Accessed: 27 July 2014].

[24] POV-Ray. "Benchmarking with POV-Ray," www.povray.org. [Online]. Available: http://www.povray.org/download/benchmark.php [Accessed: $27^{\text {th }}$ July 2014].

[25] SPEC. "SPEC CPU 2006," www.spec.org. [Online]. Available: http://www.spec.org/cpu2006/CFP2006/ [Accessed: 27 July 2014].

[26] GNU Go. "GNU Go 3.8," www.gnugo.org. [Online]. Available: http://www.gnu.org/software/gnugo/ [Accessed: $27^{\text {th }}$ July 2014].

[27] Center for Molecular Modeling and Bioinformatics. "Scalable Molecular Dynamics," www.ks.uiuc.edu. [Online]. Available: http://www.ks.uiuc.edu/Research/namd/ [Accessed: 27 July 2014].

[28] STREAM. "Sustainable Memory Bandwidth in High Performance Computers," www.cs.virginia.edu. [Online]. Available: http://www.cs.virginia.edu/stream/ [Accessed: 27th July 2014].

[29] Unixbench. "byte-unixbench," code.google.com. [Online]. Available: https://code.google.com/p/byte-unixbench/ $\quad\left[\right.$ Accessed: 30 ${ }^{\text {th }}$ October 2014].

[30] Dhrystone. "Dhrystone," www.princeton.edu. [Online]. Available: http://www.princeton.edu/ achaney/tmve/wiki100k/docs/Dhrystone.htm [ [Accessed: 30th October 2014].

[31] Whetstone. "Whetstone," en.wikipedia.org [Online]. Available: http://en.wikipedia.org/wiki/Whetstone_(benchmark) [Accessed: $30^{\text {th }}$ October 2014].

[32] Xen. "The Xen Project," www.xenproject.org. [Online]. Available: http://www.xenproject.org/ [Accessed: 30 ${ }^{\text {th }}$ October 2014].

[33] AWS. "T2 Instances," docs.aws.amazon.com. [Online]. Available: http://docs.aws.amazon.com/AWSEC2/latest/UserGuide/t2instances.html [Accessed: 30th October 2014].

[34] X. Zhang, et el, 2013, "CPI^2: CPU Performance isolation for shared compute cluster", in Proc. of the $8^{\text {th }}$ ACM European Conference on Computer Systems, pp 379-391, 2013.

[35] Openstack. “OpenStack," www.openstack.org. [Online]. Available: http://www.openstack.org/ [Accessed: 30 $0^{\text {th }}$ October 2014]. 